\title{
Myocardial viability testing: Still viable after stich?
}

\author{
Robert O. Bonow, MD, and Thomas A. Holly, $\mathrm{MD}^{\mathrm{b}}$
}

Numerous studies dating back to the 1970s have demonstrated that left ventricular (LV) systolic dysfunction in patients with coronary artery disease (CAD) is not always an irreversible process related to previous myocardial infarction (MI), as LV function may improve substantially, and even normalize, after coronary artery bypass graft (CABG) surgery. Imaging protocols with SPECT and PET tracers, dobutamine echocardiography, and contrast-enhanced cardiac magnetic resonance (CMR) have been developed specifically to target viable vs nonviable myocardium and thus identify patients with the potential for improvement in $\mathrm{LV}$ function with revascularization. Assessment of myocardial viability is often used to select patients with LV dysfunction for CABG. Following this trend, a number of studies have suggested that identification of viable myocardium will also predict which patients will have improved survival after CABG. These have been summarized in a meta-analysis ${ }^{1}$ and two subsequent reviews. $^{2,3}$

However, there are limitations of the many cohort studies that have fueled the enthusiasm for viability testing as a prerequisite for referral of patients for myocardial revascularization. The majority of such studies have been retrospective in nature, and it is not certain whether the decision for CABG may have been influenced in many cases by viability status. There was often no (or inadequate) adjustment for important baseline variables, such as age and medical comorbidities. Perhaps most importantly, these cohort studies were carried out before modern aggressive medical therapy, and in particular, therapy with beta blockers was underutilized in comparison with current medical standards. In many studies, the actual medical therapy

\footnotetext{
From the Center for Cardiovascular Innovation, Department of Medicine, ${ }^{a}$ and Division of Cardiology, Department of Medicine, ${ }^{b}$ Northwestern University Feinberg School of Medicine, Chicago, IL. Reprint requests: Robert O. Bonow, MD, Center for Cardiovascular Innovation, Department of Medicine, Northwestern University Feinberg School of Medicine, 645 North Michigan Avenue, Suite 1006, Chicago, IL 60611; r-bonow@northwestern.edu.

J Nucl Cardiol 2011;18:991-4.

1071-3581/\$34.00

Copyright (C) 2011 American Society of Nuclear Cardiology.

doi:10.1007/s12350-011-9453-1
}

employed in those who were not revascularized was not stated.

The Surgical Treatment of Ischemic Heart Failure (STICH) trial is the first prospective randomized trial testing the hypothesis that CABG improves survival in patients with ischemic LV dysfunction compared to the outcome with aggressive medical therapy. ${ }^{4,5}$ The STICH trial provided the first opportunity to assess the interaction between myocardial viability and survival in randomized patients who were all eligible for medical management alone and also eligible for CABG in addition to aggressive medical therapy. The prospective STICH viability sub-study tested the hypothesis that assessment of myocardial viability identifies patients with $\mathrm{CAD}$ and LV dysfunction who have the greatest survival benefit with $\mathrm{CABG}$ compared to aggressive medical therapy. ${ }^{6}$

Criteria for enrollment in the STICH revascularization trial of CABG vs medical therapy included coronary artery anatomy amenable for $\mathrm{CABG}$ in the absence of left main disease and a LV ejection fraction $\leq 35 \%$. All 1212 randomized patients in the STICH revascularization trial were eligible for viability testing with SPECT myocardial perfusion imaging or dobutamine echocardiography. Viability testing was optional at enrolling sites and was not a prerequisite for enrollment in STICH. The SPECT imaging protocols that were employed for assessing myocardial viability were standard, accepted protocols validated in previous clinical studies and in widespread practice. These included thallium-201 stress-redistribution-reinjection imaging, thallium-201 rest-redistribution imaging, and nitrateenhanced technetium-99m perfusion imaging. The dobutamine echocardiography protocol was the standard low-dose dobutamine protocol employed for viability testing, with staged increases in dobutamine dose, starting at a dose of $5 \mathrm{mcg} / \mathrm{kg} / \mathrm{min}$. The criteria for myocardial viability were prospective and pre-specified. For SPECT, a 17-segment model was used, and patients were considered to have myocardial viability when $\geq 11$ segments manifested viability based on relative tracer activity. It is noteworthy that this assessment of the total extent of viable myocardium with the use of SPECT is the same concept as that used in the many retrospective SPECT studies cited by Allman et $\mathrm{al}^{1}$ and Schinkel et $\mathrm{al}^{2}$ that reported improved survival in patients with viable 
myocardium who were treated with $\mathrm{CABG}$, as compared to those treated with medical therapy. For the dobutamine echocardiography studies, a 16 segment model was used, and patients were considered to have viable myocardium when there were $\geq 5$ segments with dysfunction at rest manifesting contractile reserve with dobutamine. Recognizing that these prespecified definitions of myocardial viability assessed viability in a binary fashion based on pre-specified thresholds, additional analyses were also performed in which viability scores were assessed as continuous variables and also assessed according to whether patients had viability scores above or below the median value.

The primary endpoint of the STICH viability substudy was all-cause mortality, and secondary endpoints included cardiovascular mortality and mortality plus cardiovascular hospitalization. Intention-to-treat analysis was the primary method used.

Of the 1212 patients in the STICH revascularization trial, 618 underwent a myocardial viability test and 594 did not. In 17 patients, the viability test was unusable either because it was performed outside the appropriate time window of 90 days prior to randomization or because the images were of poor quality. Thus, 601 patients (49.6\% of the total trial population) had a usable viability test. Of these, 471 were studied by SPECT and 280 by dobutamine echocardiography, with 150 patients studied by both modalities. The mean LV ejection fraction was $26.7 \%$.

Based on the prespecified definitions of viability, 487 of the 601 patients $(81 \%)$ were considered to have substantial viability, and 114 were considered nonviable. Patients with viable myocardium had fewer previous MIs, higher LV ejection fractions, and smaller LV end diastolic and end systolic volume indices (EDVI and ESVI, respectively) compared to patients with nonviable myocardium. During a median follow-up period of 5.1 years, follow-up status was ascertained in $99.6 \%$ the patients. Patients with myocardial viability had a significantly lower mortality rate compared to those without viability, with five-year mortality rates of 33\% vs $50 \%$, respectively $(P=.003)$. However, a number of other variables had a more significant impact on survival in this population, including clinical comorbidities, LV ejection fraction, and the LVEDVI and LVESVI. Hence, although myocardial viability status was a significant predictor of survival in a univariate analysis, viability was not significant when tested in a multi-variable analysis. This was true when the SPECT and dobutamine echocardiography data were combined, when the SPECT data were analyzed alone, or when the dobutamine echocardiography data were analyzed alone.

In similar fashion, analysis of the secondary endpoint of the cardiovascular mortality showed a significant relation between viability status and this endpoint in a univariate analysis, but not when subjected to the multi-variable analysis. In contrast, in both the univariate and the multi-variable analyses, the secondary endpoint of mortality plus cardiovascular hospitalization was significantly lower in patients with viability than in those without viability.

Treatment assignment to medical therapy vs CABG plus medical therapy did not demonstrate a significant interaction with viability status relative to survival. That is, both in patients with viability and those without viability, there was no significant difference in survival in those treated with medical therapy vs those treated with $C A B G$ plus medical therapy. There was also no interaction between treatment assignment and myocardial viability with respect to the secondary endpoints of cardiovascular modalities or mortality plus cardiovascular hospitalization. This was true not only for the primary analysis using the intention-to-treat approach, but also when crossover patients were accounted for and analysis was performed according to the actual treatment received.

The results of STICH run counter to the conclusions of many previous, predominantly retrospective cohort studies, and thus were not anticipated by many in the imaging community. Hence, they have engendered considerable comment. Clearly, there are limitations of the STICH trial and its analyses. As there was a lack of viability data in all patients, the data pertain only to a subpopulation of the overall STICH enrollment. Patients undergoing viability testing in STICH tended to be sicker than those without testing (as indicated in Table S2 in the supplementary appendix ${ }^{6}$ ), with more previous MIs, a higher prevalence of atrial fibrillation and previous percutaneous revascularization, higher utilization of beta blockers and ACE inhibitors, lower ejection fractions, and higher LVEDVIs and LVESVIs. Thus, the results relative to outcome with surgical vs medical therapy in such patients may not pertain to those with less severe LV dysfunction. It is noteworthy that ejection fractions have been higher in patients in most previous studies, ${ }^{1-3}$ and it is conceivable that viability testing might provide different insights in patients with less severe LV dysfunction.

The STICH analysis was limited to SPECT and dobutamine echocardiography alone, and did not include data using PET or CMR imaging methods. However, it is uncertain whether PET would provide different prognostic data compared with SPECT, as reviews and meta-analyses on this subject have not demonstrated that PET predicts survival with greater precision than with SPECT or dobutamine echocardiography. ${ }^{1-3}$ In the PET and Recovery Following Revascularization (PARR)-2 trial, ${ }^{7}$ randomization to PET viability imaging failed to 
demonstrate improved survival after $\mathrm{CABG}$ compared to standard care. In addition, Seibelink et al randomized patients to PET vs SPECT assessment of viability, after which the viability data were made available to their physicians and integrated into management decisions. ${ }^{8}$ There was no difference in survival in those studied with PET vs SPECT. Whether contrast-enhanced CMR can improve upon the results of SPECT, PET, and dobutamine echocardiography, in terms of identifying patients whose outcome is enhanced with $\mathrm{CABG}$ over medical therapy alone, is yet to be determined.

The STICH results should also not be viewed in a vacuum, as other randomized trials have also failed to demonstrate an improvement in survival in patients with viable myocardium treated with $\mathrm{CABG}$ compared to medical therapy alone. The Heart Failure Revascularisation Trial (HEART) identified patients with LV dysfunction and myocardial viability, assessed by PET, SPECT, or dobutamine echocardiography, who were randomized to conservative management vs angiography with the intent of percutaneous or surgical revascularization. ${ }^{9}$ Although the trial was terminated prematurely because of under-enrollment, the survival curves of patient treated medically and those treated with revascularization were virtually superimposable out to 5.5 years. In the viability ancillary study of the Occluded Artery Trial (OAT), patients with viable myocardium after acute MI had similar degrees of improvement in $\mathrm{LV}$ function whether treated with medical therapy or revascularization. ${ }^{10}$ These OAT results support previous studies demonstrating that viable but dysfunctional myocardium in patients with ischemic LV dysfunction may improve substantially with aggressive medical therapy, especially with the use of beta blockers. ${ }^{11-13}$ It is noteworthy that the use of beta blockers was very high in STICH, HEART, and OAT, and much higher than in the previous non-randomized reports. ${ }^{1-3}$ In this light, the mortality rates of patients with viable myocardium treated medically in STICH were considerably lower than those reported in the metaanalysis of Allman et $\mathrm{al}^{1}$.

The STICH viability sub-study represents the largest report to date relating myocardial viability to clinical outcomes of patients with CAD and LV dysfunction. Moreover, this is the first study to assess these relationships prospectively among patients who were all eligible for CABG as well as optimal medical management alone.

The STICH results do demonstrate a significant association between myocardial viability and outcome, especially with respect to the combined endpoint of survival plus cardiovascular hospitalization, which is a standard endpoint in heart failure trials. Regarding survival alone, however, the association of myocardial viability and survival was rendered nonsignificant when subjected to a multi-variable analysis that included other clinical and functional variables of prognostic value. The STICH results also fail to demonstrate a significant interaction between myocardial viability and medical vs surgical treatment with respect to mortality, whether assessed according to treatment assigned (intention-totreat) or to the treatment actually received. The STICH data indicate that in patients with CAD and severe $\mathrm{LV}$ dysfunction, assessment of myocardial viability does not identify patients who will have the greatest survival benefit from adding CABG to aggressive medical therapy. The implications are that viability testing should not be considered a prerequisite for decisions regarding medical vs surgical management in such patients. Imaging should be reserved for those patients in whom management decisions are difficult in view of age, comorbidities, or complex coronary anatomy, and in whom additional information may be necessary to guide therapy recommendations.

\section{References}

1. Allman KC, Shaw LJ, Hachamovitch R, Udelson JE. Myocardial viability testing and impact of revascularization on prognosis in patients with coronary artery disease and left ventricular dysfunction: A meta-analysis. J Am Coll Cardiol 2002;39:1151-8.

2. Schinkel AFL, Bax JJ, Poldermans D, Elhendy A, Ferrari R, Rahimtoola SH. Hibernating myocardium: Diagnosis and patient outcomes. Curr Prob Cardiol 2007;32:375-410.

3. Camici PG, Prasad SK, Rimoldi OE. Stunning, hibernation, and assessment of myocardial viability. Circulation 2008;117:103-14.

4. Velazquez EJ, Lee KL, O'Connor CM, Oh JK, Bonow RO, Pohost GM, et al. The rationale and design of the Surgical Treatment for Ischemic Heart Failure (STICH) Trial. J Thorac Cardiovasc Surg 2007;134:1540-7.

5. Velazquez EJ, Lee KL, Deja MA, Jain A, Sopko G, Marchenko A, et al. Coronary-artery bypass surgery in patients with left ventricular dysfunction. N Engl J Med 2011;364:1607-16.

6. Bonow RO, Maurer G, Lee KL, Holly TA, Binkley PF, DesvigneNickens $\mathrm{P}$, et al. Myocardial viability and survival in ischemic left ventricular dysfunction. N Engl J Med 2011;364:1617-25.

7. Beanlands RSB, Nichol G, Huszti E, Humen D, Racine N, Freeman $\mathrm{M}$, et al. F-18-fluorodeoxyglucose positron emission tomography imaging-assisted management of patients with severe left ventricular dysfunction and suspected coronary disease: A randomized, controlled trial (PARR-2). J Am Coll Cardiol 2007;50:2002-12.

8. Siebelink HM, Blanksma P, Crijns H, Bax JJ, van Boven AJ, Kingma $T$, et al. No difference in cardiac event-free survival between positron emission tomography and single-photon emission computed tomography-guided patient management. J Am Coll Cardiol 2001;37:81-8.

9. Cleland JGF, Calvert M, Freemantle N, Arrow Y, Ball SG, Bonser RS, et al. The Heart Failure Revascularisation Trial (HEART). Eur J Heart Fail 2011;13:227-33.

10. Udelson JE, Pearte CA, Kimmelstiel CD, Kruk M, Kufera JA, Forman SA, et al. The Occluded Artery Trial (OAT) Viability Ancillary Study (OAT-NUC): Influence of infarct zone viability on left ventricular remodeling after percutaneous coronary intervention versus optimal medical therapy alone. Am Heart $\mathrm{J}$ 2011;161:611-21. 
11. Bello D, Farah GM, Di Luzio S, Shah D, Parker M, Johnson MR, et al. Gadolinium cardiovascular magnetic resonance predicts reversible myocardial dysfunction and remodeling in heart failure patients undergoing beta-blocker therapy. Circulation 2003;108: 1945-53.

12. Cleland JGF, Pennell DJ, Ray SG, Coats AJ, Macfarlane PW, Murray GD, et al. Myocardial viability as a determinant of the ejection fraction response to carvedilol in patients with heart failure (CHRISTMAS trial): Randomised controlled trial. Lancet 2003;362:14-21.

13. Seghatol FF, Shah DJ, DiLuzio S, Bello D, Johnson MR, Cotts WG, et al. Relation between contractile reserve and improvement in left ventricular function with beta blocker therapy in patients with heart failure secondary to ischemic or idiopathic dilated cardiomyopathy. Am J Cardiol 2004;93:854-9. 